\title{
Accuracy of cornea and lens biometry using anterior segment optical coherence tomography
}

\author{
Mark C. M. Dunne \\ Leon N. Davies \\ James S. Wolffsohn \\ Aston University \\ Ophthalmic Research Group \\ Birmingham \\ United Kingdom
}

\begin{abstract}
We assess the accuracy of the Visante anterior segment optical coherence tomographer (AS-OCT) and present improved formulas for measurement of surface curvature and axial separation. Measurements are made in physical model eyes. Accuracy is compared for measurements of corneal thickness $\left(d_{1}\right)$ and anterior chamber depth $\left(d_{2}\right)$ using-built-in AS-OCT software versus the improved scheme. The improved scheme enables measurements of lens thickness $\left(d_{3}\right)$ and surface curvature, in the form of conic sections specified by vertex radii and conic constants. These parameters are converted to surface coordinates for error analysis. The built-in AS-OCT software typically overestimates [mean \pm standard deviation(SD) $] d_{1}$ by $+62 \pm 4 \mu \mathrm{m}$ and $d_{2}$ by $+4 \pm 88 \mu \mathrm{m}$. The improved scheme reduces $d_{1}$ $(-0.4 \pm 4 \mu \mathrm{m})$ and $d_{2}(0 \pm 49 \mu \mathrm{m})$ errors while also reducing $d_{3}$ errors from $+218 \pm 90$ (uncorrected) to $+14 \pm 123 \mu \mathrm{m}$ (corrected). Surface $x$ coordinate errors gradually increase toward the periphery. Considering the central $6-\mathrm{mm}$ zone of each surface, the $x$ coordinate errors for anterior and posterior corneal surfaces reached $+3 \pm 10$ and $0 \pm 23 \mu \mathrm{m}$, respectively, with the improved scheme. Those of the anterior and posterior lens surfaces reached $+2 \pm 22$ and $+11 \pm 71 \mu \mathrm{m}$, respectively. Our improved scheme reduced AS-OCT errors and could, therefore, enhance pre- and postoperative assessments of keratorefractive or cataract surgery, including measurement of accommodating intraocular lenses. @ 2007 Society of Photo-Optical Instrumentation Engineers. [DOI: $10.1117 / 1.2821844]$
\end{abstract}

Keywords: biometry; anterior segment optical coherence tomography; accuracy; physical model eyes.

Paper 07219RR received Jun. 15, 2007; revised manuscript received Aug. 21, 2007; accepted for publication Aug. 22, 2007; published online Dec. 18, 2007.

\section{Introduction}

The anterior segment optical coherence tomographer (ASOCT) (Visante ${ }^{\mathrm{TM}}$, Carl Zeiss Meditec, Dublin, California) has been used for anterior chamber depth measurements ${ }^{1-3}$ and phakic intraocular lens implant evaluations. ${ }^{4}$ Accurate measurements of anterior segment ocular dimensions are important for cataract surgery and keratorefractive surgery and to determine the risk of angle closure glaucoma. ${ }^{1}$ AS-OCT has built-in software to obtain corneal thickness and anterior chamber depth. ${ }^{4}$ However, we are unaware of any previous reports on (1) the accuracy of AS-OCT measurements using a model eye or (2) the measurement of cornea and lens surface curvature using this instrument. The purpose of our study was to carry out these assessments and present a new scheme for measuring axial distances and surface curvature.

\section{Methods}

The physical model eye, in which the cornea and intraocular lens were varied, was described by Kirschkamp et al. ${ }^{5}$ and has

Address all correspondence to Mark Dunne, Ophthalmic Research Group, School of Life and Health Sciences, Aston University, Birmingham B4 7ET, U.K. Tel: +44 (0) 121 204; Fax: +44 (0) 121204 4048; E-mail: m.c.m.dunne@aston.ac.uk been used for evaluation of phakometry ${ }^{6}$ and Hartmann-Shack aberrometry. ${ }^{7}$ The five variations studied included a range of corneal radii (anterior: 6.8 to $8.7 \mathrm{~mm}$; posterior: 6.3 to $8.1 \mathrm{~mm}$ ), lens radii (anterior: 7.6 to $13.6 \mathrm{~mm}$; posterior: $\quad-8.3$ to $-4.5 \mathrm{~mm})$, anterior chamber depths (1.9 to $4.1 \mathrm{~mm})$, and lens thicknesses $(3.5$ to $4.5 \mathrm{~mm})$. All model corneal thicknesses were $0.8 \mathrm{~mm}$. Model corneas and lenses were made from PMMA (refractive index 1.493) and were separated by water (refractive index 1.333).

For each model eye, three horizontal AS-OCT images, captured in the anterior segment single-image capture mode, were analyzed. The first image included corneal thickness, anterior chamber depth, and corneal surface curvature corrected using built-in AS-OCT software. The Visante software (version 1.0.12.1896) detects and fits the corneal surfaces through an edge-detection algorithm. This fit was manually optimized using the built-in AS-OCT software, particularly in the peripheral cornea where the fit was often poor. The second image included these parameters without correction. The third image involved repositioning the instrument head to enable measurement of uncorrected lens thickness and surface curvatures. The Visante software again attempts to detect the cor-

1083-3668/2007/12(6)/064023/5/\$25.00 @ 2007 SPIE 
Dunne, Davies, and Wolffsohn: Accuracy of cornea and lens biometry using anterior segment...

neal surfaces, but as they are not present, distorts the images based on the first and second surface profiles detected from the anterior plane of the image. Therefore the surface correction was removed prior to analysis.

The AS-OCT does not currently measure surface curvature. A Liberty BASIC 4.0 (Shoptalk Systems, Framingham, Massachusetts) program was written to measure coordinates of corneal and lens surfaces to subpixel precision. A pixel-tomillimeter conversion factor $\quad[1$ pixel $=22 \pm 0.1 \mu \mathrm{m}$, mean \pm standard deviation $(\mathrm{SD})]$ was determined by measuring the diameter of the recess in which model corneas were held. Twenty $x$ and $y$ coordinates were determined for each surface using a cursor. The $x$ coordinate ( $\left.x_{\text {original }}\right)$ and grayscale value $(G)$ of the pixel on which the cursor was centered were retrieved. Subpixel analysis involved sampling the grayscale profile of a vertical line of pixels centered on the cursor. These pixels were assigned a location value $(L)$ ranging from -5 ( 5 pixels above the cursor) to +5 ( 5 pixels below). The peak of the gray-scale profile was calculated and used to adjust $x$ [Eq. (1)]. Note that Eq. (1) works only for the edges of model corneas and lenses that appeared as a narrow band.

$$
x_{\text {adjusted }}=x_{\text {original }}+\sum(L G) / \sum G .
$$

Conic sections were derived from surface coordinates using Eq. (2) in which $r_{0}$ is the vertex radius, and $p$ is the conic constant that determines whether the surface becomes flatter $(p<1)$ or steeper $(p>1)$ in the periphery. ${ }^{8}$ A second-order polynomial fitted to $y^{2}$ plotted as a function of $x$ yielded coefficients for $x\left(=2 r_{0}\right)$ and $x^{2}(=-p)$ :

$$
y^{2}=2 r_{0} x-p x^{2}
$$

Our comparison of measured to known model eye dimensions involved three steps. In step 1, calculations were carried out to add optical distortion, due to refraction at the corneal and lenticular surfaces, to the known model eye dimensions. This step ensured that instrument distortion correction factors were transferable to any human eye. Having completed this step, any remaining discrepancy between known and measured eye dimensions was assumed to be due to instrument distortion. In step 2, averaged instrument distortion correction factors were derived for each eye component. Step 3 involved carrying out calculations (effectively the reverse of those carried out in step 1) to correct optical distortion.

Optical distortion was added to known model eye dimensions, in step 1, using well-known paraxial ray tracing formulas. These calculations made use of refractive indices corrected for OCT light wavelength ${ }^{4}(1.310 \mu \mathrm{m})$. Refractive indices were corrected using Eq. (3) in which $n_{\lambda}$ is the refractive index at wavelength $\lambda$, and $n$ is the refractive index for white light. ${ }^{9}$

$$
n_{\lambda}=n+0.0512-0.1455 \lambda+0.0961 \lambda^{2} .
$$

Averaged instrument correction factors, derived in step 2, for axial distances were calculated by dividing optically distorted axial distances, calculated in step 1 , by the corresponding distances measured in uncorrected AS-OCT images. For surface curvature, optically distorted surface $x$ coordinates were di-

\begin{tabular}{|c|c|c|}
\hline Component & Correction Factor & Equation Numbe \\
\hline \multicolumn{3}{|l|}{ Surface curvature } \\
\hline anterior cornea & $\begin{array}{l}r_{1} x l=r_{1} x U(1.5582-0.0037 y \\
\left.+0.0025 y^{2}\right)\end{array}$ & (5) \\
\hline posterior cornea & $\begin{array}{l}r_{2} x l=r_{2} x U(1.5454-0.0012 y \\
\left.+0.0020 y^{2}\right)\end{array}$ & (6) \\
\hline anterior lens & $\begin{array}{l}r_{3} x l=r_{3} x U(1.8412+0.0501 y \\
\left.-0.0519 y^{2}\right)\end{array}$ & (7) \\
\hline posterior lens & $\begin{array}{l}r_{4} x I=r_{4} x U(1.5373+0.0036 y \\
\left.-0.0035 y^{2}\right)\end{array}$ & (8) \\
\hline \multicolumn{3}{|l|}{ Axial distances } \\
\hline corneal thickness & $d_{1} l=d_{1} U(0.6350)$ & (9) \\
\hline anterior chamber & $\begin{array}{l}d_{2} l=d_{2} U\left(0.9002-0.0848 d_{2} U\right. \\
\left.+0.0314 d_{2} U^{2}\right)\end{array}$ & (10) \\
\hline lens thickness & $\begin{array}{l}d_{3} l=d_{3} U\left(28.184-13.634 d_{3} U\right. \\
\left.+1.7017 d_{3} U^{2}\right)\end{array}$ & (11) \\
\hline
\end{tabular}
vided by uncorrected $x$ coordinates, at fixed values of $y$.
Table 1 AS-OCT instrument distortion correction factors.

These were derived from measurements on each model eye but are transferable to any human eye. Equations (5) to (8) convert surface $x$ coordinates measured from uncorrected AS-OCT images $\left(r_{1-4 x} U\right)$, at any value of $y$, to values corrected for instrument distortion $\left(r_{1-4 x} l\right)$. Equations (9) to (11) convert axial distances measured from uncorrected AS-OCT images $\left(d_{1-3} U\right)$ to values corrected for instrument distortion $\left(d_{1-3} l\right)$

Model eye dimensions dictated the fixed $y$ values used for corneas (from 0.5 to $5.0 \mathrm{~mm}$ in $0.5-\mathrm{mm}$ steps) and lenses (from 0.5 to $3.0 \mathrm{~mm}$ in 0.5 - $\mathrm{mm}$ steps). Surface $x$ coordinates were calculated using

$$
x=r_{0}-\left(r_{0}^{2}-p y^{2}\right)^{0.5} / p .
$$

The instrument distortion correction factor for the anterior corneal surface was determined using three steel ball bearings. The diameters of these were measured with Precision Gold electronic digital callipers (Maplin Electronics, South Yorkshire, England) and converted to radii of curvature; $7.49 \pm 0.005, \quad 7.94 \pm 0.003, \quad$ and $9.52 \pm 0.004 \mathrm{~mm}$ (mean $\pm 95 \%$ confidence interval). Table 1 shows the correction factors [Eqs. (5) to (11)] that were derived as described above and which are transferable to any human eye.

Before applying paraxial formulas to correct optical distortion in step 3 , it was necessary to convert the surface $x$ coordinates corrected for instrument distortion $\left(r_{1-4} x I\right)$ into local sagittal radii of curvature $\left(r_{1-4 s} I\right)$. This was achieved by fitting second-order polynomial curves to the square of the fixed $y$ coordinates $\left(y^{2}\right)$ plotted as a function of the surface $x$ coordinates $\left(r_{1-4} x I\right)$ to calculate vertex radii $\left(r_{1-40} I\right)$ and conic constants $\left(p_{1-4} I\right)$. The values $r_{10} I$ and $p_{1} I$ represented the fully corrected anterior corneal surface vertex radius $\left(r_{10}\right)$ and conic constant $\left(p_{1}\right)$ as this surface was viewed in air. Equation (12) was then used to calculate sagittal radii of curvature $\left(r_{2-3 s} I\right)$ for each fixed $y$ coordinate. ${ }^{10}$

$$
r_{s}=\left[r_{0}^{2}+(1-p) y^{2}\right]^{0.5} \text {. }
$$


Table 2 shows the paraxial formulae [Eqs. (13 to (45)] used to correct optical distortion, which are transferable to any human eye. These calculations were repeated for sagittal radii of curvature at each fixed $y$ coordinate.

Finally, a scheme described by Douthwaite ${ }^{11}$ was adopted to derive optically corrected vertex radii $\left(r_{2-40}\right)$ and conic constants $\left(p_{2-4}\right)$ from the optically corrected sagittal radii $\left(r_{2-4 s}\right)$ calculated for each fixed $y$ coordinate, as described in Table 2. This involved plotting $r_{s}^{2}$ (on the $y$ axis) against $y^{2}$ (on the $x$ axis) to produce a straight line for which, according to Eq. (46), the intercept is $r_{o}^{2}$ and the slope is $(1-p)$.

$$
r_{s}^{2}=r_{0}^{2}+(1-p) y^{2}
$$

\section{Results}

Figure 1 shows the profiles and axial separations and Table 3 the errors of uncorrected and corrected measurements made in all five model eyes. Uncorrected AS-OCT measurements overestimate cornea and lens thickness while underestimating anterior chamber depth and surface curvature. Correction with built-in AS-OCT software substantially reduced these errors, but greater accuracy was achieved with the new scheme.

\section{Discussion}

AS-OCT is a noncontact procedure capable of providing a comprehensive evaluation of anterior segment biometry. While there have been several reports on the use of model eyes to assess biometric accuracy, that of Dubbelman et al. ${ }^{12}$ using corrected Scheimpflug images is of particular relevance as it also covered the entire anterior segment with a noncontact technique. Their typical levels of accuracy were similar to ours for corneal thickness ( 1 compared to $0.4 \mu \mathrm{m})$ and lens thickness (44 compared to $14 \mu \mathrm{m}$ ). After conversion of their surface radius errors to $x$ coordinate errors at $y=3 \mathrm{~mm}$, their errors for the anterior cornea ( 2 compared to $3 \mu \mathrm{m}$ ), posterior cornea ( 1 compared to $0 \mu \mathrm{m}$ ), anterior lens ( 2 compared to $2 \mu \mathrm{m}$ ), and posterior lens ( 4 compared to $11 \mu \mathrm{m}$ ) were also comparable with ours. Interestingly, anterior corneal surface errors of both imaging techniques fall within clinically acceptable limits ${ }^{13}$ of $0.02 \mathrm{~mm}$ (corresponding to $x$ coordinate errors of $2 \mu \mathrm{m}$ at $y=3 \mathrm{~mm}$ ).

Kashiwagi et al. ${ }^{14}$ used a model eye to assess the accuracy of their scanning peripheral anterior chamber depth analyzer and found errors of up to $1.8 \%$. This equates to errors of up to $70 \mu \mathrm{m}$ and is, again, comparable with those of our study ( $98 \mu \mathrm{m}$ based on two SDs from the mean).

Barry et al. ${ }^{6}$ used different variations of the model eye described in our study to assess the accuracy of a Purkinje imaging method and found that anterior and posterior lens surface radii could be measured to an accuracy of 0.02 to $0.10 \mathrm{~mm}$ and 0.10 to $0.55 \mathrm{~mm}$, respectively; equivalent to $x$ coordinate errors (at $y=3 \mathrm{~mm}$ ) of 1 to 5 and 16 to $96 \mu \mathrm{m}$, respectively. These errors are a little higher than those of Scheimpflug and AS-OCT imaging techniques. A more detailed comparison between Scheimpflug and Purkinje imaging methods has been described elsewhere. ${ }^{15}$

Paraxial ray tracing equations were used to demonstrate that these produce results to acceptable levels of accuracy. Exact ray-tracing formulas may further improve accuracy but
Table 2 Paraxial ray tracing formulas [Eqs. (13) to (45)] used to correct sagittal radii of curvature $\left(r_{2-4 s} l\right)$ and axial distances $\left(d_{1-3} l\right)$ for optical distortion for each fixed $y$ coordinate.

Equation Equation Number

Surface vertex powers (based on vertex radii $r_{10} l, r_{20} l$, and $r_{30} l$ )

$F_{10} l=\left[1000\left(n_{2}-1\right)\right] / r_{10} l$

$F_{20} l=\left[1000\left(n_{3}-n_{2}\right)\right] / r_{20} l$

$F_{30} l=\left[1000\left(n_{4}-n_{3}\right)\right] / r_{30} l$

Ray trace for optically corrected corneal thickness $\left(d_{1}\right)$

$L_{1}=1000 / d_{1} I$

$L_{1}{ }^{\prime}=L_{1}+F_{10}$

$d_{1}=\left(1000 n_{2}\right) / L_{1}^{\prime}$

Ray trace for optically corrected posterior corneal sagittal radius of curvature $\left(r_{2 s}\right)$

$L_{1}=1000 /\left(d_{1} l+r_{2 s} l\right)$

$L_{1}{ }^{\prime}=L_{1}+F_{10}$

$r_{2 s}=\left[\left(1000 n_{2}\right) / L_{1}{ }^{\prime}\right]-d_{1}$

Ray trace for optically corrected anterior chamber depth $\left(d_{2}\right)$

$$
\begin{aligned}
& L_{1}=1000 /\left(d_{1} l+d_{2} l\right) \\
& L_{1}{ }^{\prime}=L_{1}+F_{10} l \\
& L_{2}=L_{1}{ }^{\prime} /\left\{1-\left[d_{1} /\left(1000 n_{2}\right)\right] L_{1}{ }^{\prime}\right\} \\
& L_{2}{ }^{\prime}=L_{2}+F_{20} l \\
& d_{2}=\left(1000 n_{3}\right) / L_{2}{ }^{\prime}
\end{aligned}
$$

Ray trace for optically corrected anterior lens sagittal radius of curvature $\left(r_{3 s}\right)$

$$
\begin{aligned}
& L_{1}=1000 /\left(d_{1} I+d_{2} I+r_{3 s} l\right) \\
& L_{1}{ }^{\prime}=L_{1}+F_{10} I \\
& L_{2}=L_{1}{ }^{\prime} /\left\{1-\left[d_{1} /\left(1000 n_{2}\right)\right] L_{1}{ }^{\prime}\right\} \\
& L_{2}{ }^{\prime}=L_{2}+F_{20} l \\
& r_{3 s}=\left[\left(1000 n_{3}\right) / L_{2}{ }^{\prime}\right]-d_{2}
\end{aligned}
$$

Ray trace for optically corrected lens thickness $\left(d_{3}\right)$

$$
\begin{aligned}
& L_{1}=1000 /\left(d_{1} I+d_{2} I+d_{3} l\right) \\
& L_{1}{ }^{\prime}=L_{1}+F_{10} l \\
& L_{2}=L_{1}{ }^{\prime} /\left\{1-\left[d_{1} /\left(1000 n_{2}\right)\right] L_{1}{ }^{\prime}\right\} \\
& L_{2}{ }^{\prime}=L_{2}+F_{20} l \\
& L_{3}=L_{2}{ }^{\prime} /\left\{1-\left[d_{2} /\left(1000 n_{3}\right)\right] L_{2}{ }^{\prime}\right\} \\
& L_{3}{ }^{\prime}=L_{3}+F_{30} l \\
& d_{3}=\left(1000 n_{4}\right) / L_{3}{ }^{\prime}
\end{aligned}
$$


Dunne, Davies, and Wolffsohn: Accuracy of cornea and lens biometry using anterior segment...

Table 2 (Continued.)

Equation

Equation Number

Ray trace for optically corrected posterior lens sagittal radius of curvature $\left(r_{4 s}\right)$

$L_{1}=1000 /\left(d_{1} l+d_{2} l+d_{3} l+r_{4 s} l\right)$

$L_{1}{ }^{\prime}=L_{1}+F_{10} l$

$L_{2}=L_{1}{ }^{\prime} /\left\{1-\left[d_{1} /\left(1000 n_{2}\right)\right] L_{1}{ }^{\prime}\right\}$

$L_{2}^{\prime}=L_{2}+F_{20} I$

$L_{3}=L_{2}^{\prime} /\left\{1-\left[d_{2} /\left(1000 n_{3}\right)\right] L_{2}{ }^{\prime}\right\}$

$L_{3}{ }^{\prime}=L_{3}+F_{30}{ }^{\prime}$

$r_{4 s}=\left[\left(1000 n_{4}\right) / L_{3}{ }^{\prime}\right]-d_{3}$

Refractive indices $\left(n_{2}\right.$ to $n_{4}$ ) were corrected for AS-OCT light wavelength using Eq. (3) (see text). These equations are transferable to any human eye.

at the loss of convenience; clinicians can program spread sheets with the paraxial equations shown in Table 2 rather than having to purchase specialist software for exact ray tracing.

We were concerned that instrument distortion correction factors for corneal thickness might not be transferable to human eyes as all model eyes had a fixed corneal thickness of $0.80 \mathrm{~mm}$. To test this, we made further measurements on five contact lenses with the same anterior surface radii $(7.8 \mathrm{~mm})$ and refractive indices (1.493) but with central thicknesses of between 0.3 and $0.7 \mathrm{~mm}$ in $0.1-\mathrm{mm}$ steps. Corneal thickness instrument correction factors and errors for these did not differ from those shown in Tables 1 and 3 .

Although the formulas shown in Table 1 adequately correct instrument distortion, exploration of the possible sources of

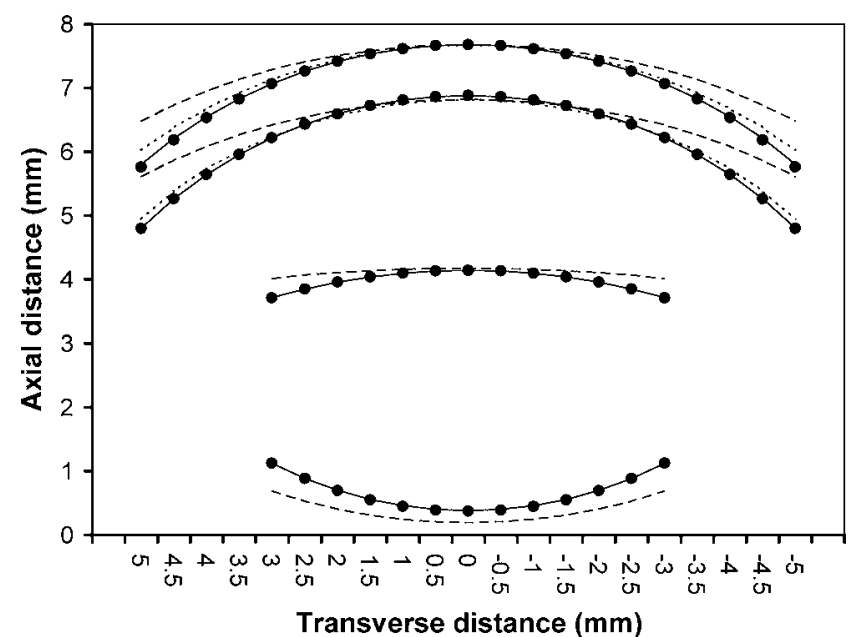

Fig. 1 Profiles and axial separations of actual surfaces (solid lines) compared to uncorrected measured surfaces (dashed lines) and those corrected using built in AS-OCT software (dashed lines) and the equations shown in Tables 1 and 2 (circles). Each profile represents the average of the five model eyes tested.
Table 3 Errors (mean $\pm \mathrm{SD}, \mu \mathrm{m}$ ) for ocular components measured in five model eyes using AS-OCT with and without correction using inbuilt software and the new scheme.

Corrected

\begin{tabular}{llcl} 
Component & Uncorrected & AS-OCT Software & New Scheme \\
\hline Corneal thickness & $+60 \pm 0$ & $+62 \pm 4$ & $-0.4 \pm 4$ \\
Anterior chamber depth & $-96 \pm 111$ & $+4 \pm 88$ & $0 \pm 49$ \\
Lens thickness & $+218 \pm 90$ & - & $+14 \pm 123$ \\
Anterior corneal surface & $-221 \pm 33$ & $-68 \pm 15$ & $3 \pm 10$ \\
Posterior corneal surface & $-264 \pm 69$ & $-88 \pm 22$ & $0 \pm 23$ \\
Anterior lens surface & $-264 \pm 70$ & - & $+2 \pm 22$ \\
Posterior lens surface & $-246 \pm 213$ & - & $+11 \pm 71$ \\
\hline
\end{tabular}

Coordinate errors are shown for corneal and lens surfaces. As surface $x$ coordinate errors gradually increased toward the periphery, these are only shown for $y$ coordinate values of $3 \mathrm{~mm}$. This represents the maximum errors expected when sampling surfaces over a zone of $6 \mathrm{~mm}$ diameter, i.e., similar to corneal ablation zones in keratorefractive surgery or optic zone diameters of intraocular lenses. Positive values represent overestimated axial distances or surface curvature.

this distortion was beyond the scope of this study. Such exploration would also require commercially sensitive information about the instrument's design.

In conclusion, the improved computing scheme reduces the errors of the built-in AS-OCT software and enables measurement of surface curvature in the form of vertex radii and conic constants. Further, the equations of our new scheme are made available for readers to use in their own clinics and laboratories. The accuracy of this technique is comparable to that of other noncontact techniques. Surface curvatures can be measured to within clinically acceptable limits over zones of at least $6 \mathrm{~mm}$ diameter, i.e., similar to optic zone diameters of intraocular lenses and corneal ablation zones in keratorefractive surgery. ${ }^{16}$

\section{References}

1. R. Lavanya, L. Teo, D. S. Friedman, H. T. Aung, M. Baskaran, H. Gao, T. Alfred, S. K. Seah, K. Kashiwagi, P. J. Foster, and T. Aung, "Comparison of anterior chamber depth measurements using the IOLMaster, scanning peripheral anterior chamber depth analyser and anterior segment optical coherence tomography," Br. J. Ophthamol. 91(8), 1023-1026 (2007).

2. G. Nemeth, A. Vajas, A. Tsorbatzoglou, B. Kolozsvari, L. Modis, Jr., and A. Berta, "Assessment and reproducibility of anterior chamber depth measurement with anterior segment optical coherence tomography compared with immersion ultrasonography," J. Cataract Refractive Surg. 33(3), 443-447 (2007).

3. G. Baïkoff, H. Jitsuo Jodai, and G. Bourgeon, "Measurement of the internal diameter and depth of the anterior chamber: IOLMaster versus anterior chamber optical coherence tomographer," J. Cataract Refractive Surg. 31(9), 1722-1728 (2005).

4. G. Baïkoff, "Anterior segment OCT and phakic intraocular lenses: a perspective," J. Cataract Refractive Surg. 32(11), 1827-1835 (2006).

5. T. Kirschkamp, M. Jöckel, G. Wählisch, and J. C. Barry, "Konstruktion eines Modellauges zur Simulation von Purkinje-Spiegelbildern für die Bestimmung der Krümmungsradien und der Lage der Augenlinse," Biomed. Tech. 43, 318-325 (1998). 
6. J. C. Barry, M. C. M. Dunne, and T. Kirschkamp, "Phakometric measurement of ocular surface radius of curvature and alignment: evaluation of method with physical model eyes," Ophthalmic Physiol. Opt. 21(6), 450-460 (2001).

7. A. Cervino, S. L. Hosking, and M. C. M. Dunne, "Operator induced errors in Hartmann-Shack wavefront sensing: model eye study," J. Cataract Refractive Surg. 33(1), 115-121 (2007).

8. M. C. M. Dunne, "A computing scheme for determination of retinal contour from peripheral refraction, keratometry and A-scan ultrasonography," Ophthalmic Physiol. Opt. 15(2), 133-143 (1995).

9. H. L. Liou and N. A. Brennan, "An anatomically accurate, finite schematic eye for optical modelling," J. Opt. Soc. Am. A 14(3), 1684-1695 (1997)

10. A. G. Bennett, "Aspheric and continuous curve contact lenses. Part three," Optomet. Today 28, 238-242 (1988).

11. W. A. Douthwaite, "EyeSys corneal topography measurement applied to calibrated ellipsoid convex surfaces," Br. J. Ophthamol. 79(9),
797-801 (1995).

12. M. Dubbelman, R. G. van der Heijde, and H. A. Weeber, "The thickness of the aging human lens obtained from corrected Scheimpflug images," Optom. Vision Sci. 78(6), 411-416 (2001).

13. P. M. Monteiro and C. C. Hull, "The effect of videokeratoscope faceplate design on radius of curvature maps," Ophthalmic Physiol. Opt. 27(1), 76-84 (2007).

14. K. Kashiwagi, F. Kashiwagi, Y. Toda, K. Osada, T. Tsumura, and S. A. Tsukahara, "A newly developed peripheral anterior chamber depth analysis system: principle, accuracy, and reproducibility," $\mathrm{Br}$. J. Ophthamol. 88(8), 1030-1035 (2004).

15. P. Rosales, M. Dubbelman, S. Marcos, and R. van der Heijde, "Crystalline lens radii of curvature from Purkinje and Scheimpflug imaging," J. Vision 6(10), 1057-1067 (2006).

16. S. Marcos, "Aberrometry: basic science and clinical applications," Bull. Soc. Belge Ophtalmol 302, 197-213 (2006). 